\title{
Report of Neuropathic Pain After Dental Implant Placement: A Case Series
}

\author{
Constantinus Politis, MD, DDS, MHA, MM, $\mathrm{PhD}^{1} /$ Jimoh Agbaje, BDS, DMD, FMCDS, MMI, $\mathrm{PhD}^{2}$ / \\ Jeroen Van Hevele, MD, DDS, OMF ${ }^{3}$ /Laura Nicolielo, DDS, MSc ${ }^{4}$ /Antoon De Laat, DDS, $\mathrm{PhD}^{5}$ / \\ Ivo Lambrichts, DDS, $\mathrm{PhD}^{6} /$ Reinhilde Jacobs, DDS, MSc, $\mathrm{PhD}, \mathrm{Dhc}^{7}$
}

\begin{abstract}
Purpose: To report on a cohort of patients referred to a tertiary center because of neuropathic pain after dental implant placement. Materials and Methods: This retrospective study of pain after dental implant placement involved a minimum follow-up of 12 months after the initial diagnosis of neuropathic pain or persistent, uncontrolled postoperative pain at the Department of Oral and Maxillofacial Surgery, Leuven University, Leuven, Belgium, from January 2013 to June 2014. Results: Following clinical and radiologic examination, the cause of pain was established in 17 of 26 patients, while the cause was unknown in 9 of 26 patients. Regular implants were placed in the mandibles of 18 patients; in the remaining 8 patients, 6 received regular implants and 2 received a zygoma implant in the maxilla. Surgical management alone brought relief to 2 patients, surgical and pharmacologic management did so for 12 patients, and pharmacologic management alone brought relief for 10 patients. Conclusions: Early removal of an at-risk implant seems justified, preferably within 48 hours after placement. No treatment, either surgical or medical, seems to cure neuropathic pain, but amitriptyline appears to be associated with consistent improvement in symptoms. INT J ORAL MaXILlofac ImPLANTS 2017;32:439-444. doi: 10.11607/jomi.5241
\end{abstract}

Keywords: antidepressant, dental implant, inferior alveolar nerve, nerve pain, nerve regeneration, neuropathic pain

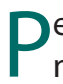
ersistent, uncontrollable postoperative pain and neuropathic pain are disabling iatrogenic conditions following surgical procedures, and they can have severe medical, economic, and psychologic implications. The incidence varies from $6 \%$ to about $85 \%$ after

\footnotetext{
${ }^{1}$ OMFS-IMPATH Research Group, Department of Imaging and Pathology; Professor and Head, Department of Oral and Maxillofacial Surgery, KULeuven, Leuven, Belgium, and Leuven University Hospitals, Belgium.

${ }^{2}$ Postdoctoral Fellow, OMFS-IMPATH Research Group, Department of Imaging and Pathology, KULeuven, Leuven, Belgium.

${ }^{3}$ Resident, Oral and Maxillofacial Surgery, Leuven University Hospitals, Leuven, Belgium.

${ }^{4} \mathrm{PhD}$ Student, University of Leuven, Leuven, Belgium.

${ }^{5}$ Professor and Head, Department of Oral Health Sciences, Leuven University Hospitals, Leuven, Belgium.

${ }^{6}$ Professor Faculty of Medicine, Hasselt University, Diepenbeek, Belgium.

${ }^{7}$ Professor, OMFS-IMPATH Research Group, Department of Imaging and Pathology, KULeuven, Leuven, Belgium.
}

Correspondence to: Dr Constantinus Politis, Professor and Head, Department of Oral and Maxillofacial Surgery, KULeuven, Kapucijnenvoer 7 blok a-box 7001, 3000 Leuven, Belgium. Email: constantinus.politis@uzleuven.be

@2017 by Quintessence Publishing Co Inc. amputation. ${ }^{1}$ In the trigeminal nerve distribution, this condition is rare, with an incidence probably between $0.6 \%$ and $5 \% .^{2,3}$ The reason for these differences is not well understood. In the orofacial area, most preoperative and postoperative sensory impulses are mediated through the trigeminal nerve, whereas elsewhere in the body, non-nerve tissues such as visceral structures are usually involved. Why the trigeminal nerve system is so resistant to neuropathic pain or persistent uncontrollable pain is unknown, but the range of published incidence rates indicates that its occurrence is underreported.

Nerve damage in oral and maxillofacial surgery is mainly the result of third molar surgery, followed by local anesthesia, endodontic treatment, orthognathic surgery, dental implant surgery, and intraoral treatment of other pathologic conditions. Nerve damage after third molar surgery or local anesthesia in the mandible can affect both the lingual and inferior alveolar nerves, but nerve damage in endodontic treatment or after dental implant placement affects the inferior alveolar nerve almost exclusively. ${ }^{4}$

Neural damage is characterized by loss or gain in sensation (negative or positive symptoms), as well as other pain conditions (eg, neuropathic pain)., 3 The negative symptoms (sensory deficits) present as anesthesia or hypoesthesia, and the positive symptoms present as paresthesias, dysesthesias, and hyperesthesia among others. ${ }^{3,5}$ 
Nerve compression due to edema, entrapment, or the effect of chemicals such as local anesthetics or irrigation fluid used during endodontic treatment causes a transient inhibition of nerve function that produces the symptoms of hypoesthesia, anesthesia, or muscle weakness (if the motor nerve is involved). ${ }^{6-8}$ Removing the cause of entrapment, relieving edema, and wearing off of chemical effects restore the functionality of the affected nerve; this is different from what is observed in orthognathic cases in which the nerve can be elongated or bruised, or in oral pathology in which the tumor may be in close apposition to the nerve or may even invade it. Placement of implants on nerves or branches of the nerve results in persistent symptoms; removal of such implants may fail to relieve the symptom of nerve damage if not done early. 6,9

Previous studies show nerve recovery in more than $80 \%$ of patients with hypoesthesia, with the worst recovery seen in patients with neuropathic pain. ${ }^{10}$

The incidence of neurovascular complications and neuropathic pain after implant placement is believed to be $0 \%$ to $24 \%$ for transient damage and $0 \%$ to $11 \%$ for permanent damage. ${ }^{11,12,13}$ Indeed, neuropathic pain in the maxillofacial area was reported only in 1993 by MacMahon. ${ }^{14}$ Its definition was revised in 2011. ${ }^{15}$ The contemporary definition of neuropathic pain by the International Association of the Study of Pain is "pain caused by a lesion or disease of the somatosensory nervous system."

It is possible to think that in dental implant surgery, the primary lesion is always easy to find; however, that is not the case, and this can interfere with categorizing the pain as neuropathic or persistent nonresponding postoperative pain. Because the definition of neuropathic pain involves a postoperative nerve lesion or dysfunction after dental implant placement, it is popularly defined as iatrogenic, whereas it is defined as idiopathic if no such nerve damage can be detected.

The caseload of referred patients with neuropathic or persistent postoperative pain seen between January 2013 and June 2014 included 65 patients, most of whom were affected after dental implant placement (31 patients), followed by orthognathic surgery (10), oral pathology conditions or surgery for oral pathology (5), trauma (5), third molar surgery (3), local anesthesia (3), non-third molar extraction (3), infection (2), endodontics (2), and preprosthetic placement (1). This distribution clearly differentiates the incidence of neuropathic pain, which is primarily found after dental implant surgery, from the incidence of inferior alveolar nerve damage, which is primarily found after third molar surgery.

Because neuropathic pain after dental implant placement is reported only rarely, the descriptive analysis of the caseload of a tertiary referral center merits the attention of the dental and maxillofacial surgical community. However, it is difficult to accurately calculate the incidence after dental implant placement. In Belgium, about 55,000 to 60,000 dental implants are placed yearly in fewer than 30,000 patients. An oral survey of legally recognized pain clinics in Belgium showed that most patients with postoperative neuropathic or idiopathic pain after dental implant surgery were also referred to tertiary maxillofacial clinics. On the basis of the small number of tertiary oromaxillofacial surgery clinics, the best estimate of neuropathic pain incidence is $0.1 \%$ to $0.5 \%$ among patients after dental implant surgery. The purpose of this study is to report the occurrence of neuropathic pain after dental implant placement in a cohort of patients referred to a tertiary center.

\section{MATERIALS AND METHODS}

Patients who visited the Department of Oral and Maxillofacial Surgery, Leuven University, Leuven, Belgium, from January 2013 to June 2014 and met the inclusion criteria were retrospectively selected for this study. The inclusion criteria consisted of a diagnosis of persistent, uncontrolled postoperative pain or neuropathic pain after dental implant placement in the mandible or maxilla or in the zygomatic area, with a minimum follow-up of 12 months after first diagnosis of neuropathic pain or persistent, uncontrolled postoperative pain. A total of 31 patients met the inclusion criteria. No patients were excluded because follow-up was available either by direct examination or telephone contact with one of the authors (J.V.H.). This retrospective study was approved by the Medical Ethical Committee of Leuven University Hospitals (number S57587). Surgical procedures for pain management included implant removal, apicectomy, nerve decompression by means of unilateral sagittal split osteotomy, and cryotherapy. The relief of pain symptoms in this study is defined as patient satisfaction with the level of pain control to the point that no additional method of pain control is needed.

\section{RESULTS}

The study included 26 patients (10 men, 16 women) with an age range of 19 to 75 years and a mean age of $55.9 \pm 11.9$ years. During the $1 \frac{1}{2}$-year study period, all patients presented to the oral and maxillofacial unit of UZ Leuven Hospital with neuropathic pain after dental implant placement.

After clinical and radiologic examination, the cause of pain was established in 17 of 26 patients (patients with evidence of nerve trauma) while the cause was 


\section{Table 1 General Information About Patients and Implants}

\begin{tabular}{|c|c|c|c|c|c|}
\hline & & & $\begin{array}{l}\text { Group } 1(n=17) \\
\text { Physical trauma }\end{array}$ & $\begin{array}{c}\text { Group } 2(n=9) \\
\text { No physical trauma }\end{array}$ & $\begin{array}{c}\text { Total } \\
(n=26)\end{array}$ \\
\hline \multirow[t]{4}{*}{ Location of implant } & Maxilla & Anterior & 3 & 3 & 6 \\
\hline & & Posterior & 3 & 5 & 8 \\
\hline & Mandible & Anterior & 3 & 2 & 5 \\
\hline & & Posterior & 9 & 4 & 13 \\
\hline \multirow[t]{2}{*}{ Type of implant } & & Regular & 15 & 9 & 24 \\
\hline & & Zygoma & 2 & 0 & 2 \\
\hline
\end{tabular}

\section{Table 2 Characteristics of Pain and Pain Onset After Implant Placement}

\begin{tabular}{|c|c|c|c|c|}
\hline & & $\begin{array}{l}\text { Group } 1(\mathrm{n}=17) \\
\text { Physical trauma }\end{array}$ & $\begin{array}{c}\text { Group } 2(n=9) \\
\text { No physical } \\
\text { trauma }\end{array}$ & Total $(n=26)$ \\
\hline \multirow[t]{5}{*}{ Type of pain } & Burning sensation & 3 & 3 & 6 \\
\hline & Continuous & 3 & 1 & 4 \\
\hline & Electrical/sharp & 1 & 2 & 3 \\
\hline & Sensibility loss & 9 & 0 & 9 \\
\hline & Unknown & 3 & 1 & 4 \\
\hline \multirow[t]{2}{*}{ Location } & Localized & 10 & 3 & 13 \\
\hline & Radiated & 2 & 7 & 9 \\
\hline \multirow[t]{5}{*}{ Period of pain onset } & Immediately ( $<24$ hours after placement) & 12 & 5 & 17 \\
\hline & 2 days & 1 & 0 & 1 \\
\hline & After loading & 0 & 2 & 2 \\
\hline & After 2 months & 0 & 1 & 1 \\
\hline & Unknown & 3 & 2 & 5 \\
\hline \multirow{4}{*}{$\begin{array}{l}\text { Period of presentation } \\
\text { at center after implant } \\
\text { placement }^{a}\end{array}$} & 2-12 months & 5 & 0 & 5 \\
\hline & 13-23 months & 1 & 2 & 3 \\
\hline & $2-13$ years & 4 & 5 & 9 \\
\hline & Unknown & 4 & 0 & 4 \\
\hline
\end{tabular}

${ }^{a}$ Cases in which patients received implants at another center.

unknown in 9 of 26 patients (no obvious evidence of trauma to a nerve). Table 1 gives an overview of the type and location of implants in the two groups of patients. In 18 patients, regular implants were placed in the mandible. Of the remaining 8 patients, 6 received regular implants and 2 received a zygoma implant in the maxilla.

Table 2 shows the pain characteristics (type and location) and the period of onset after implant placement. Nine patients presented with somatosensory loss, while 4 patients described their pain as continuous. The onset of pain was less than 24 hours after implant placement in 17 patients, 12 of whom had evidence of trauma to the nerve. In 2 patients, the pain started after implant loading, and in 5 patients, the onset of pain could not be established.

Figure 1 shows the distribution of patients among different treatment modalities. Surgery was the first line of treatment in patients with an obvious cause of nerve injury (12 patients). If surgical treatment did not improve symptoms or if surgical treatment was not an option, pharmacologic treatment was administered. Amitriptyline in combination with vitamins (cyanocobalamin) was the first line of treatment for patients receiving pharmacologic intervention.

Surgical treatment alone brought pain relief in 2 patients, surgical and pharmacologic treatment brought relief in 12 patients, and pharmacologic treatment alone brought relief in 10 patients. Figures 2 and 3 show the varying surgical and pharmacologic treatments and corresponding numbers of patients with pain relief. Improvement in 8 of 15 patients was observed with amitriptyline and cyanocobalamin. Carbamazepine treatment was administered in 3 patients and yielded improvement in 1 . Duloxetine led to symptom improvement in 1 of 3 patients, while pregabalin and baclofen resulted in no pain improvement in any of the patients treated with these medications. 


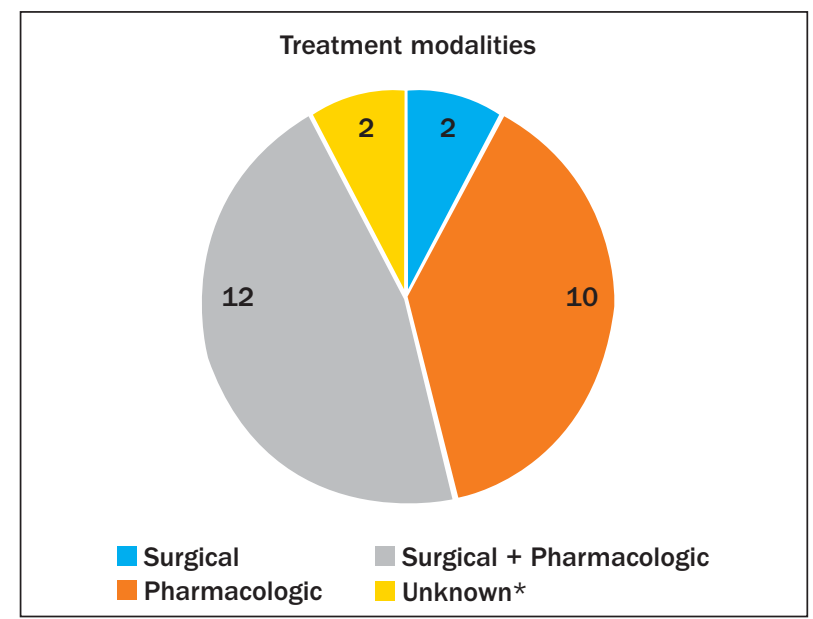

Fig 1 Number of patients according to treatment modality. * Patients who did not return to the clinic or did not follow the treatment regimen.

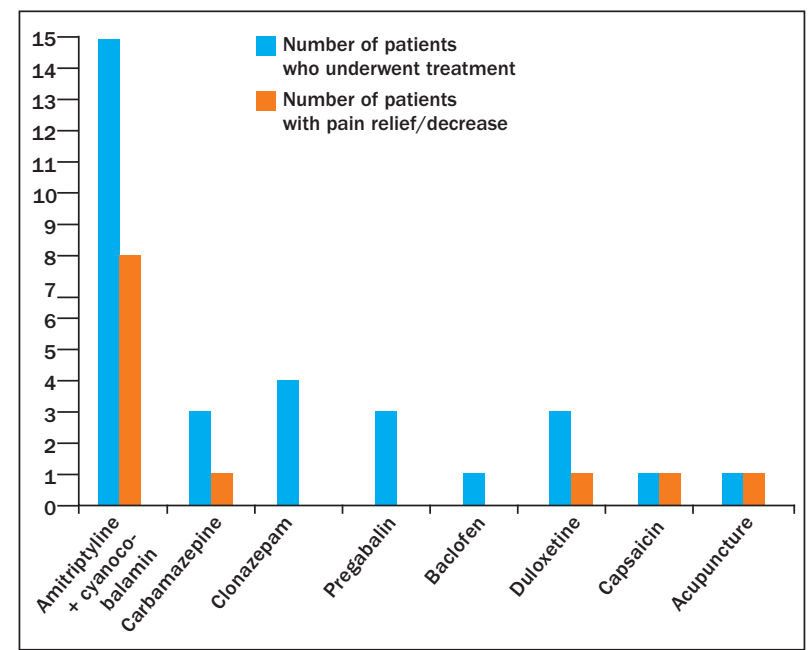

Fig 3 Pharmacologic management of patients with neuropathic pain.

\section{DISCUSSION}

Patients with neuropathic pain present with constant pain that can be superficial or deep, sharp or aching, or lancinating (ie, sudden, sharp, and severe bursts of pain); allodynia (ie, pain experienced after normally nonpainful stimuli, such as a light touch) is also a possibility. The discomfort is usually chronic, and the patient may describe it as a burning sensation; a sharp, stabbing, or shooting pain; or a pain similar to an electric shock. ${ }^{3,5}$

Some patients report somatosensory loss in the affected region. Other characteristics of neuropathic pain include a lack of response to anti-inflammatory

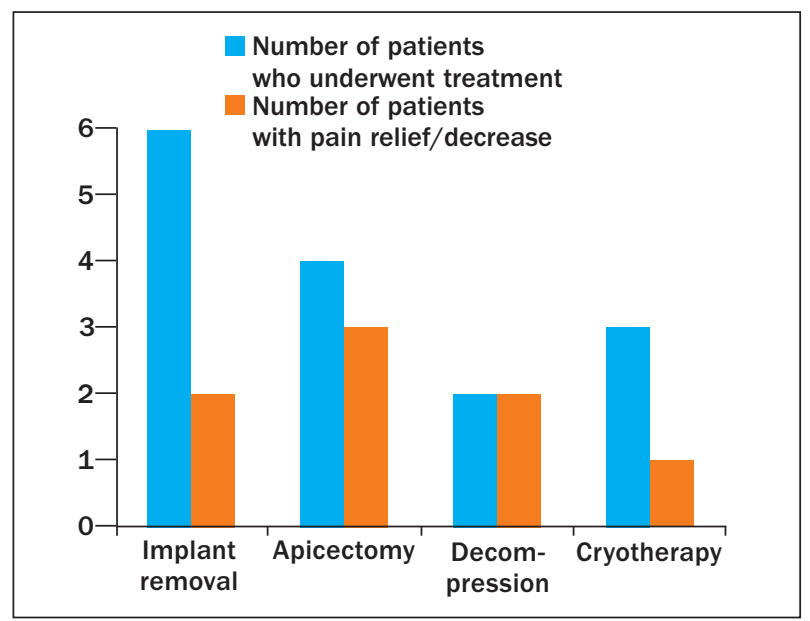

Fig 2 Surgical management of patients with neuropathic pain.

pain killers (nonsteroidal anti-inflammatory drugs [NSAIDs], paracetamol), improved symptoms in the morning, minimal sleep disturbance, and worsening symptoms during the day or accompanying stress, fatigue, and illness. ${ }^{5}$

In this study, immediate pain or pain within 24 hours after implant placement or loading was reported by 18 of 26 patients who presented with neuropathic pain. Trauma is a known causative factor in neuropathic pain, 5,11,16,17 and injury to the nerve may result in persistent orofacial pain (Fig 4). Patients present with a range of symptoms of nerve damage depending on the extent of trauma; those with a laceration of the inferior alveolar nerve are at greater risk of developing neuropathic pain. ${ }^{18}$ Pain within 24 hours after implant placement or loading should be investigated and treated because it can be a telltale sign of nerve damage.

The age of patients without obvious evidence of trauma to the nerve ranged from 51 to 75 years, with a mean age of 62 years. The age range for patients with evidence of trauma was 19 to 73 years, and their mean age was 56 years. Age older than 45 years has been implicated as a predisposing factor for developing neuropathic pain. ${ }^{5}$ Most patients in this study were older than 45 years. The persistent pain in this patient group could have been the result of age combined with a procedure involving compression or partial severance of the inferior alveolar nerve or complete discontinuity of the lingual nerve with a proximal stump neuroma, ${ }^{5}$ as seen in those with evidence of trauma. In addition, it is pertinent that most recovery, especially after surgical and pharmacologic intervention, was observed in patients with obvious evidence of trauma. 
Also important to note is that late surgical intervention after onset of neuropathic pain did little to improve the patient's condition. ${ }^{3}$ Most patients in this study were referred by other centers after developing neuropathic pain. As a result, implant removal after onset of pain (within 3 months) was possible in only 2 of the 26 patients, and these two patients experienced obvious improvement. Implants in 4 other patients were removed 4 months after placement, without obvious pain improvement. Early repair of nerve injury has been identified as the most critical factor in the surgical treatment and prevention of neuropathic pain. ${ }^{3,19}$

Implants were removed in patients with obvious trauma (Fig 2). Other surgical treatments included apicectomy of the implant, decompression of the nerve by means of unilateral sagittal split osteotomy, or cryotherapy. If surgical treatment did not result in pain relief or if surgical treatment was not an option, pharmacologic treatment was administered. ${ }^{20,21}$ Neuropathic pain treatment remains unsatisfactory despite a substantial increase in the number of trials conducted. ${ }^{22}$ The use of low-dose antidepressants (amitriptyline, nortriptyline) can be effective for symptomatic relief. ${ }^{23}$ To date, no pharmacologic treatment has been effective in completely resolving patients' neuropathic pain. Topical agents, such as capsaicin, were advised for one patient, who achieved pain relief after using it. Another patient reported improvement after undergoing acupuncture.

Management of pain should be tailored to each patient on the basis of pain type, the causative disease, and psychosocial factors. ${ }^{5}$ An accurate preoperative patient history is advised, as is administration of neuropathic pain medication immediately postoperatively in patients with nerve damage before pain is well established. ${ }^{3,18}$ In addition, clinicians should perform patient profiling and identify risk factors for developing neuropathic pain in all patients scheduled for implant surgery. Proper localization of the inferior alveolar nerve before implant placement is also an essential preventive step. $^{5}$

At-risk patients are those who are not pain free in the region of implantation, those who experience an incapacitating pain at a certain implant site immediately after placement that does not respond to painkillers (paracetamol, NSAID, and morphine), those in whom the implant penetrates a neurovascular canal, or a combination of the preceding. Early removal of an at-risk implant seems justified, preferably within 48 hours after placement. No treatment, surgical or medical, has been shown to cure neuropathic pain, and amitriptyline seems to be the only medication associated with consistent improvement in symptoms.

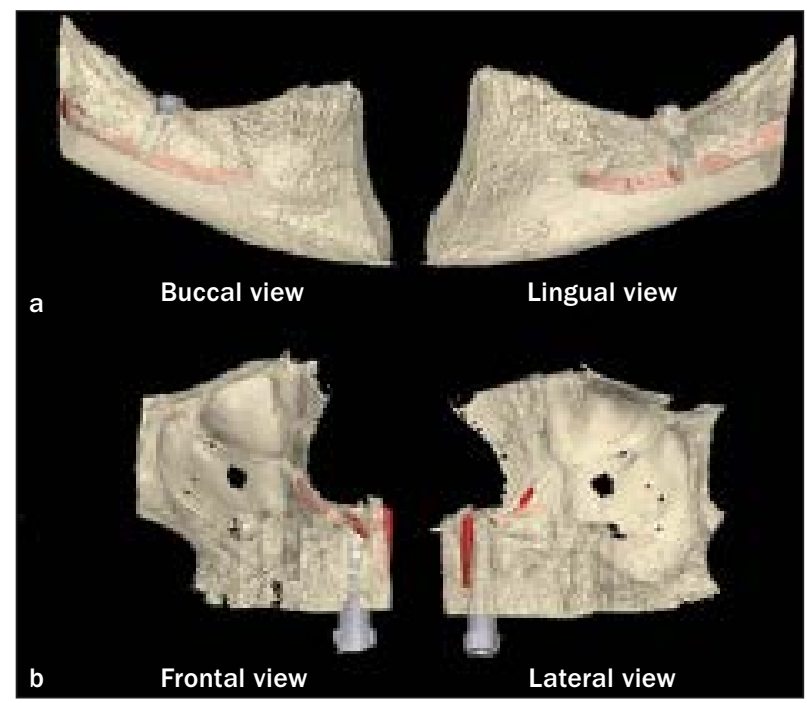

Fig 4 Three-dimensional models generated from cone beam computed tomographic scans of two cases showing the relationship between the implant (gray area) and neurovascular content (red area). (a) Mandibular alveolar canal. (b) Canalis sinuosus.

\section{CONCLUSIONS}

Management of persistent pain after placement of dental implants should be tailored to each patient on the basis of the pain type, the causative disease, and psychosocial aspects. Early removal of an at-risk implant seems justified, preferably within 48 hours after placement. No surgical or medical treatment seems to cure neuropathic pain, but amitriptyline seems to be associated with consistent improvement in symptoms.

\section{ACKNOWLEDGMENTS}

The authors reported no conflicts of interest related to this study.

\section{REFERENCES}

1. Macrae WA. Chronic pain after surgery. Br J Anaesth 2001;87:88-98

2. Jacobs R, Quirynen M, Bornstein MM. Neurovascular disturbances after implant surgery. Periodontol 2000;66:188-202.

3. Politis C, Lambrichts I, Agbaje JO. Neuropathic pain after orthognathic surgery. Oral Surg Oral Med Oral Pathol Oral Radiol 2014;117:e102-e107.

4. Hillerup S. Iatrogenic injury to the inferior alveolar nerve: Etiology, signs and symptoms, and observations on recovery. Int J Oral Maxillofac Surg 2008;37:704-709.

5. Agbaje JO, Lambrichts I, Jacobs R, Politis C. Neuropathic pain after bilateral sagittal split osteotomy: Management and prevention. Plast Aesthet Res 2015;2:171-175.

6. Al-Sabbagh M, Okeson JP, Khalaf MW, Bhavsar I. Persistent pain and neurosensory disturbance after dental implant surgery: Pathophysiology, etiology, and diagnosis. Dent Clin North Am 2015;59:131-142. 
7. Tay $A B$, Zuniga JR. Clinical characteristics of trigeminal nerve injury referrals to a university centre. Int J Oral Maxillofac Surg 2007;36:922-927.

8. Ziccardi VB, Assael LA. Mechanisms of trigeminal nerve injuries. Atlas Oral Maxillofac Surg Clin North Am 2001;9:1-11.

9. Wijbenga JG, Verlinden CR, Jansma J, Becking AG, Stegenga $B$. Long-lasting neurosensory disturbance following advancement of the retrognathic mandible: Distraction osteogenesis versus bilateral sagittal split osteotomy. Int J Oral Maxillofac Surg 2009;38:719-725

10. Agbaje JO, Van de Casteele E, Hiel M, Verbaanderd C, Lambrichts I, Politis C. Neuropathy of trigeminal nerve branches after oral and maxillofacial treatment. J Maxillofac Oral Surg 2016;15(3):321-327.

11. Boogaard S, de Vet HC, Faber CG, Zuurmond WW, Perez RS. An overview of predictors for persistent neuropathic pain. Expert Rev Neurother 2013;13:505-513.

12. Al-Sabbagh M, Okeson JP, Khalaf MW, Bhavsar I. Persistent pain and neurosensory disturbance after dental implant surgery: Pathophysiology, etiology, and diagnosis. Dent Clin North Am 2015;59:131-142.

13. Agbaje JO, Sun Y, Vrielinck L, Schepers S, Lambrichts I, Politis C. Risk factors for the development of lower border defects after bilateral sagittal split osteotomy. J Oral Maxillofac Surg 2013;71:588-596.

14. MacMahon SH. TMJ dysfunction: The surgical dilemma. Br J Oral Maxillofac Surg 1993;31:336-337.

15. Jensen TS, Baron R, Haanpää M, et al. A new definition of neuropathic pain. Pain 2011;152:2204-2205.
16. Agbaje JO, Salem AS, Lambrichts I, Jacobs R, Politis C. Systematic review of the incidence of inferior alveolar nerve injury in bilateral sagittal split osteotomy and the assessment of neurosensory disturbances. Int J Oral Maxillofac Surg 2015;44:447-451.

17. Boogaard S, Heymans MW, Patijn J, et al. Predictors for persistent neuropathic pain: A Delphi survey. Pain Physician 2011;14:559-568.

18. Kuhlefelt $M$, Laine $P$, Suominen $A L$, Lindqvist $C$, Thorén $H$. Nerve manipulation during bilateral sagittal split osteotomy increases neurosensory disturbance and decreases patient satisfaction. J Oral Maxillofac Surg 2014;72:2052-2055.

19. Costigan M, Scholz J, Woolf CJ. Neuropathic pain: A maladaptive response of the nervous system to damage. Annu Rev Neurosci 2009;32:1-32.

20. Haythornthwaite JA, Benrud-Larson LM. Psychological assessment and treatment of patients with neuropathic pain. Curr Pain Headache Rep 2001;5:124-129.

21. Marchiori ÉC, Barber JS, Williams WB, Bui PQ, O'Ryan FS. Neuropathic pain following sagittal split ramus osteotomy of the mandible: Prevalence, risk factors, and clinical course. J Oral Maxillofac Surg 2013;71:2115-2122.

22. Attal N, Cruccu G, Haanpaa M, Haanpää M, et al. EFNS guidelines on pharmacological treatment of neuropathic pain. Eur J Neurol 2006;13:1153-1169.

23. Zin CS, Nissen LM, Smith MT, O'Callaghan JP, Moore BJ. An update on the pharmacological management of post-herpetic neuralgia and painful diabetic neuropathy. CNS Drugs 2008;22:417-442. 